\title{
Towards Semantic Social Networks
}

\author{
Jason J. Jung ${ }^{1}$ and Jérôme Euzenat ${ }^{2}$ \\ ${ }^{1}$ Department of Computer and Information Engineering, Inha University \\ Incheon, Republic of Korea 402-751 \\ ${ }^{2}$ INRIA Rhône-Alpes \& LIG, Montbonnot, France \\ j2jung@intelligent.pe.kr, Jerome.Euzenat@inrialpes.fr
}

\begin{abstract}
Computer manipulated social networks are usually built from the explicit assertion by users that they have some relation with other users or by the implicit evidence of such relations (e.g., co-authoring). However, since the goal of social network analysis is to help users to take advantage of these networks, it would be convenient to take more information into account. We introduce a threelayered model which involves the network between people (social network), the network between the ontologies they use (ontology network) and a network between concepts occurring in these ontologies. We explain how relationships in one network can be extracted from relationships in another one based on analysis techniques relying on this network specificity. For instance, similarity in the ontology network can be extracted from a similarity measure on the concept network. We illustrate the use of these tools for the emergence of consensus ontologies in the context of semantic peer-to-peer systems.
\end{abstract}

\section{Introduction}

Social networks, i.e., networks based on the relation between people is our common environment. Many have realized that social networks are a key facilitator for collaborating. Social scientists have been analyzing the effectiveness of of these networks by helping characterizing the key individuals that must be touched in order to achieve some goals [1]. Recently, on the semantic web, social networks are very often described through FOAF or FOAF-like schemata. They could as well be built upon shared address books.

However, when communities are really different or when people do not want to describe explicitly their relationships, social networks have to be inferred from secondary sources. These secondary sources are often documents: bibliometrics has for long made a speciality of inferring social networks and many other clues from databases of coauthoring and citations. The web is a rich source of documents that can be used as secondary sources for inferring social networks from analyzing the hyperlinked structure on the web [2] (along the way used by google for inferring most authoritative web pages) to exploiting the more intimate personal infospheres made of web pages, weblogs, and so on. 
Most of these sources are based on explicit links that still need from document authors (or web page designers) to know each others. In this paper, we investigate the dual principles of:

- using the knowledge structure used by individuals in order to infer some of their social relationships, and

- taking advantage of this inferred social structure in order to help people sharing their knowledge.

For that purpose, we introduce a structure made of three superposed networks that are assumed to be strongly linked:

Social network relating people on the basis of common interest;

Ontology network relating ontologies on the basis of explicit import relationships or implicit similarity;

Concept network relating concepts on the basis of explicit ontological relationships or implicit similarity.

We call this stack of interlinked networks a semantic social network.

The top-down links between these networks are obvious: people use ontologies that defines or refer to concepts. The less obvious aspect that is illustrated here is bottomup inference of relationships from one network to another. These relationships can be inferred by analyzing the structure of knowledge.

Once the structure of knowledge allows to infer "intellectual" relationships between people, it is possible to use it for helping people managing their knowledge. For instance, finding that similar people use similar ontologies or the same ontology makes them candidates to be standardized. In addition, when starting some collaboration between partners in order to merge their knowledge or to define one common ontology, it is better to start from the ontology used by considering social features such as centrality and authority.

The remainder of the paper is organized as follows. Sect.2 provides a description of a target application which uses network analysis in order to help people sharing ontology annotated resources. Sect. 3 provides the basic knowledge in social network analysis. As the main contribution, Sect. 4 and 5 explain the three-layered semantic social network structure. Sect.4 4 considers each layer, the kind of relationships it expresses and the tools that can be used for manipulating these networks. Sect. 5 investigates the relationships between these layers and the opportunity this provides to lift network structure from one layer to another. Sect. 6 presents an experiment we have conducted. Finally, in Section 7, this work is compared with previous studies related to social networks on semantic web.

\section{Emerging Collaboration in Peer to Peer Networks}

We apply semantic social networks in the context of peer-to-peer (P2P) sharing of annotations in which individuals can use ontologies for annotating resources (in our case, photographs). Because, these individuals can have different needs and different standpoints, they are not constrained to use the same ontology (contrary to BibSter [5], for 
instance). They can take advantage of standard ontologies on the web (like FOAF or EXIF), but they can extend these ontologies to their needs.

However, when people want to query other peers, for benefiting from better annotation or from other pictures, the network of heterogeneous ontologies must be dealt with. This ontology mismatch is solved by offering an alignment infrastructure to the peers that allows them to find some alignments between their ontologies and to process queries through mediators [6].

Current work on ontology matching consists of either automatically matching two ontologies without regard to its context or environment, or of interactively helping some knowledge engineer to match two ontologies. Our goal is to take into account the social networking context in order to improve the alignment results and, in turn, the social networking expertise.

In this context, semantic social network analysis has two purposes:

- helping people to find other peers with similar interests, and

- helping peers to find the best company for starting designing consensus ontologies.

For the first purpose, there are two options. On the one hand, the peers can use social network analysis (SNA) to find in their explicit personal network of peers with some the same interests. Such people would certainly use ontologies that are not totally remote and trying to match these ontologies promise to be easier and more rewarding. On the other hand, if one peer has no relation with known peers sharing the same interest, but is looking for some pictures, it will be more convenient that he looks for peers having similar ontologies. The peers can use metrics on the ontology network in order to find ontologies which are close to theirs. The underlying assumption is that these peers will have similar interest to theirs even if they are not explicitly recorded as connected. Moreover, if the metric is compatible with some matching algorithm, the matching will be easier to perform. So, in this situation, the social network is used for satisfying the need of some user (finding interesting annotated resources) and this action will contribute strengthening the social network by exhibiting new links between peers. As a simple example, Fig. 11illustrates the potential connection between unknown users by ontology alignment in a semantic social network.

Another contribution of SNA to this application is to identify the central (authoritative) ontologies at the ontology level such that aligning with these ontologies allows to be connected with the maximum number of peers (and thus retrieve the maximum number of answers).

Matching algorithms can take advantage of SNA in order to help find the closest ontologies with regards to the connectivity of users and to identify which users are more prone to the use of a pivotal ontology.

Beside matching ontologies in an ad-hoc manner, there is time when peers can imagine benefiting from putting their experience together and building some consensus ontologies. To that extent, they can also use SNA in order to identify the community (cohesive subgroups on the social level), the best peer for proposing consensus (centrality on the social network), or the ontologies which could be the seed for this collaboration process (centrality on the ontology layer). Of course, when both coincide (i.e., when the central individuals use a central ontology), the chances of success are higher. 


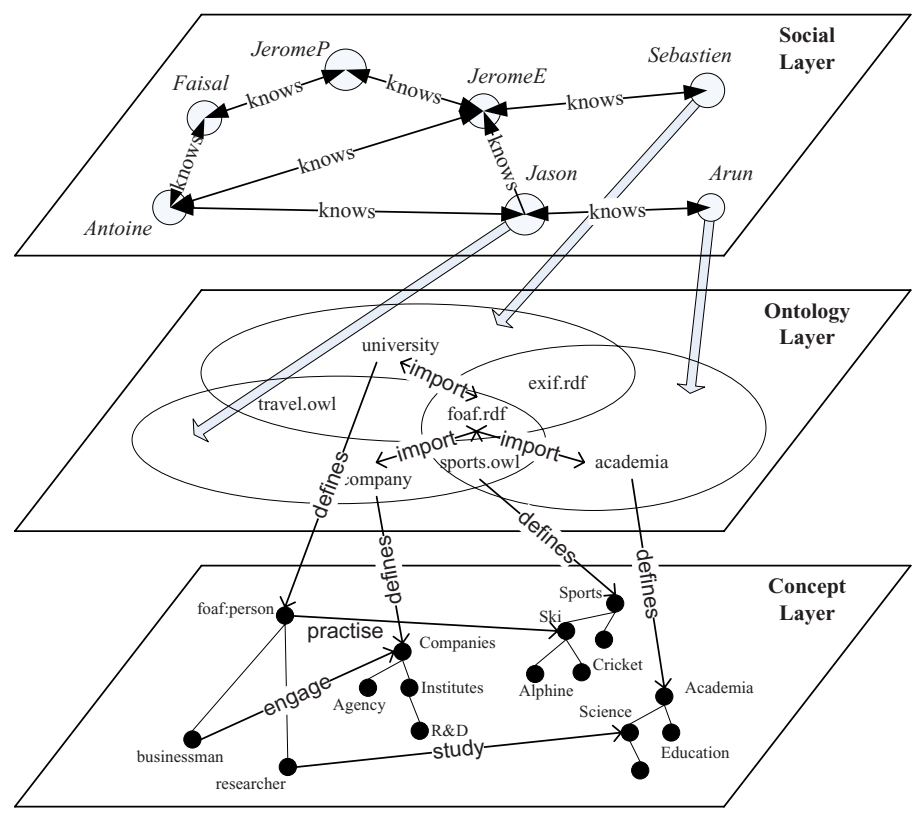

Fig. 1. A three-layered social semantic network

\section{Network Analysis}

Network analysis is based on the analysis of the relationships within a population. We here consider networks with several different relations between individuals. So, a network is characterized as a set of objects (or nodes) and a set of relations.

Definition 1 (Network). A network $\left\langle N, E^{1}, \ldots E^{n}\right\rangle$ is made of a set $N$ of nodes and $n$ sets of object pairs $E^{i} \subseteq N \times N$ the set of relations between these nodes.

We will below freely use the relations as functions $\left(E^{i}(n)=\left\{n^{\prime} \in N \mid\left\langle n, n^{\prime}\right\rangle \in E^{i}\right\}\right)$. As usual, a path $p$ between node $e$ and $e^{\prime}$ in the graph of $E^{i}$ is a sequence of edges $\left\langle e_{0}, e_{1}\right\rangle,\left\langle e_{1}, e_{2}\right\rangle, \ldots,\left\langle e_{k-1}, e_{k}\right\rangle$ from $E^{i}$ in which $e_{0}=e$ and $e_{k}=e^{\prime}$. The length of a path is its number of edges (here $k), s p^{i}\left(e, e^{\prime}\right)$ is the set of shortest paths between $e$ and $e^{\prime}$ and the shortest path distance $s p d^{i}\left(e, e^{\prime}\right)$ between two nodes $e$ and $e^{\prime}$ is the length of the shortest paths between them, when it exists. By convention, $\operatorname{spd}(e, e)=0$. Thus, $\operatorname{spd}($ Arun, Sebastien) in Fig. 11 is three via Jason and JeromeE.

Social network analysis [1] has considered various measures on the networks between people (note that these measures apply only if the network is connected)]:

Closeness. The inverse of average length of the shortest path between a node $e$ and any other node in the network:

$$
\operatorname{Closeness}^{i}(e)=\frac{|N-1|}{\sum_{e^{\prime} \in N} \operatorname{spd}^{i}\left(e, e^{\prime}\right)}
$$

\footnotetext{
${ }^{1}$ These measures are often normalized (between 0 and 1) but we present their simplest form.
} 
Betweenness. [7] The proportion of shortest paths between two nodes which contains a particular node (this measures the power of this node):

$$
\text { Betweenness }^{i}(e)=\sum_{e^{\prime}, e^{\prime \prime} \in N} \frac{\left|\left\{p \in s p^{i}\left(e^{\prime}, e\right), p^{\prime} \in s p^{i}\left(e, e^{\prime \prime}\right) \mid p \cdot p^{\prime} \in s p^{i}\left(e^{\prime}, e^{\prime \prime}\right)\right\}\right|}{\left|s p^{i}\left(e^{\prime}, e^{\prime \prime}\right)\right|}
$$

Hub and authority. There are different but interrelated patterns of power: Authorities that are referred to by many and hubs that refers to many. The highest authorities are those which are referred to by the highest hubs and the highest hubs that those which refers to the highest authorities. Kleinberg [2] proposes an iterative algorithm to measure authority and hub degree of each node in interlinked environment. Given initial authority and hub degrees of 1 , the degrees are iteratively computed by

$$
H u b_{t+1}^{i}(e)=\sum_{e^{\prime}:\left\langle e, e^{\prime}\right\rangle \in E^{i}} A u t h_{t}^{i}\left(e^{\prime}\right) \text { and } A u t h_{t+1}^{i}(e)=\sum_{e^{\prime}:\left\langle e^{\prime}, e\right\rangle \in E^{i}} H u b_{t}^{i}\left(e^{\prime}\right)
$$

Similarly to betweenness, the hub weight indicates the structural position of the corresponding user. It is a measure of the influence that people have over the spread of information through the network.

Our purpose being the identification of individual that are prone to collaborate with each others, we would like to find these clusters of individuals in the network. There are no standard method for extracting so-called cohesive subgroups in social network analysis. Many different methods are proposed based on graph-theoretic terms (e.g., cliques [1]) or clustering methods (e.g., [8]).

Definition 2 (Distance network). A distance network $\left\langle N, E^{1}, \ldots, E^{n}\right\rangle$ is made of a set $N$ of nodes and $n$ sets of distance functions $E^{i}: N \times N \longrightarrow\left[\begin{array}{ll}0 & 1\end{array}\right]$ defining the distance between nodes (so satisfying symmetry, positiveness, minimality, and triangular inequality).

Distance values can also be seen a weights or costs. It is clear that any network is a distance network which attributes either 0 or 1 as a distance. The definitions of SNA mentioned above can be adapted to distance networks if each time the cardinality of a set of edges if used, it is replaced by the sum of its distances. The distance of a path is obtained by summing the distances of its edges. One extension that must be made is to use the distance between nodes to reduce their influence to others in the computation of authority and hub degrees:

$$
H u b_{t+1}^{i}(e)=\sum_{e^{\prime} \in N} \frac{A u t h_{t}^{i}\left(e^{\prime}\right)}{E^{i}\left(e, e^{\prime}\right)} \text { and } A u t h_{t+1}^{i}(e)=\sum_{e^{\prime} \in N} \frac{H u b_{t}^{i}\left(e^{\prime}\right)}{E^{i}\left(e^{\prime}, e\right)}
$$

\section{Three-Layered Architecture for Semantic Social Networks}

In order to uncover the links between people from those that can be found from their knowledge, we propose the three-layered architecture for constructing the semantic social network. As shown in Figure 1 it consists of i) a social network $(\mathcal{S})$, ii) an ontology network $(\mathcal{O})$, and iii) a concept network $(\mathcal{C})$. The characteristics of each layer and the relationships between layers are described below. 


\subsection{Social Layer}

In the social layer $(\mathcal{S})$, nodes are representing people, and relations are the connections between peoples. A social network $\mathcal{S}$ is a directed graph $\left\langle N_{S}, E_{S}^{k n o w s}\right\rangle$, where $N_{S}$ is a set of person and $E_{S}^{k n o w s} \subseteq N_{S} \times N_{S}$ the set of relations between these persons. In most current applications, the relation used by SNA is the knows relation that can be found in FOAF.

Table 1. Closeness, authoritative and hub weights in Fig.1

\begin{tabular}{|c|c|c|c|c|c|c|c|}
\hline Weights & Arun(AS) & Antoine(AZ) & Faisal(FAK) & JeromeE(JE) & Jason $(\mathrm{JJ})$ & JeromeP(JP) & Sebastien(SL) \\
\hline Closeness & 0.5 & 0.67 & 0.5 & 0.6 & 0.67 & 0.46 & 0.4 \\
\hline Authoritative & 0.21 & 0.45 & 0.37 & 0.69 & 0.243 & 0.236 & 0.13 \\
\hline Hub & 0.01 & 0.52 & 0.27 & 0.32 & 0.54 & 0.42 & 0.275 \\
\hline
\end{tabular}

From the social network in Fig. 1, the authoritative and hub weights of three users are shown in Table 1. Obviously, the highest hub weight is assigned to Jason because he is an important and unavoidable role of bridging between the rest of users.

\subsection{Ontology Layer}

The ontology network $\mathcal{O}$ is a network $\left\langle N_{O}, E_{O}^{i}\right\rangle$, in which $N_{O}$ is a set of ontologies and $E_{O}^{i} \subseteq N_{O} \times N_{O}$ the relationships between these ontologies. There can be two main kinds of relations at this stage:

import when some ontology explicitly import another ontology;

refer when some ontology uses some concept defined in another ontology.

The objective relationship from the $\mathcal{S}$ to the $\mathcal{O}$ is through the explicit usage of an ontology by a user which can be expressed by a relation: $U s e \subseteq N_{S} \times N_{O}$.

We can easily interpret the hubs as being the ontologies that combine a large number of other ontologies. These would be an interesting starting point for any newcomer willing to annotate a similar set of objects as his friend. Likewise, authorities will be ontologies that are extended and imported by many different actors (i.e., de facto standard ontologies).

There is a difference between ontology networks and social networks though: while in social networks it is normal to be connected to several authorities, an ontology will only import one ontology on some topic. It would thus be useful to recognize those hubs that connects authorities on the same topics, these "ontologies" are likely to be the expression of an alignment between the two authorities.

\subsection{Concept Layer}

In the concept layer $(\mathcal{C})$, nodes are concepts, and links are the numerous kinds of links that can be found in ontologies. The concept network $\mathcal{C}$ is a network $\left\langle N_{C}, E_{C}^{i}\right\rangle$, in which $N_{C}$ is a set of entity of an ontology (classes, properties, individuals) and $E_{C}^{i} \subseteq$ 
$N_{C} \times N_{C}$ the relationships between these entities. These relationships are far more numerous than in the other layers and depends on the kind of entity considered. If we restrict our attention to classes, the:

subClass linking a class to its subclasses;

superClass (=subClass ${ }^{-1}$ ) linking a class to its super classes;

sibbling linking a class to its siblings;

disjoint linking a class to the classes it is explicitly disjoint with;

property (=domain $\left.{ }^{-1}\right)$ linking a class to its properties;

range $^{-1}$ linking a class to the properties that refer to it.

The objective relationship from the $\mathcal{O}$ to the $\mathcal{C}$ is through the definition of concept in an ontology which can be expressed by a relation: Defines $\subseteq N_{O} \times N_{C}$. However, this notion of definition is not easy to catch: it could be based on either the assertion of a constraint on some ontology entity or the namespace in which entity belongs. We will consider the namespace in the following.

We are here further away from social networking. As noted in [4], the notions of hub and authority cannot be understood in the same way for all the relations expressed in $\mathcal{C}$.

\section{Inferring Relationships}

This three-level semantic social network does not bring in itself new improvement for our P2P sharing application. In order to provide new insight in the possible collaborations it is necessary to analyze these networks and to propagate information from one layer to another. We explain how, starting from the lower concept layer, it is possible to enrich the upper ontology and social layers with new relations from which SNA helps finding relevant peers.

\subsection{Similarity on the Concept Layer}

Beside the numerous relationships that can be found by construction of the concept layer, new relationships can be inferred between the entities. One particular relationship that will be interesting here is similarity. In order, to find relationship between concepts from different ontologies, identifying the entities denoting the same concept is a very important feature. As a matter of fact, most of the matching algorithms use some similarity measure or distance in order to match entities.

Similarity on the concept layer can be obtained by various means [9]. Some distances can be established from the local features of entities. For instance, the name of entities can be the basis for matching them. Many techniques have been developed for comparing strings, based on their structures (like edit distance), their morphology (through lemmatization), their entry in lexicons (using WordNet). Another kind of similarity can be established based on set of shared instances like in [3].

Some other distances, more in the spirit of network analysis, can be defined from the structure of the network. For instance, [10], defines possible similarities (e.g., Sim $_{C}$, $\operatorname{Sim}_{R}, \operatorname{Sim}_{A}$ ) between classes, relationships, attributes, and instances. Given a pair of 
classes from two different ontologies, the similarity measure $\operatorname{Sim}_{C}$ is assigned in $[0,1]$. The similarity $\left(\operatorname{Sim}_{C}\right)$ between $c$ and $c^{\prime}$ is defined as

$$
\operatorname{Sim}_{C}\left(c, c^{\prime}\right)=\sum_{E \in \mathcal{N}(C)} \pi_{E}^{C} \operatorname{MSim}_{Y}\left(E(c), E\left(c^{\prime}\right)\right)
$$

where $\mathcal{N}(C) \subseteq\left\{E^{1} \ldots E^{n}\right\}$ is the set of all relationships in which classes participate (for instance, subclass, instances, or attributes). The weights $\pi_{E}^{C}$ are normalized (i.e., $\left.\sum_{E \in \mathcal{N}(C)} \pi_{E}^{C}=1\right)$.

If we consider class labels $(L)$ and three relationships in $\mathcal{N}(C)$, which are superclass $\left(E^{s u p}\right)$, subclass $\left(E^{s u b}\right)$ and sibling class $\left(E^{s i b}\right)$, Equ. 5 is rewritten as:

$$
\begin{aligned}
\operatorname{Sim}_{C}\left(c, c^{\prime}\right) & =\pi_{L}^{C} \operatorname{sim}_{L}\left(L\left(A_{i}\right), L F\left(B_{j}\right)\right) \\
& +\pi_{\text {sup }}^{C} M \operatorname{Sim}_{C}\left(E^{\sup }(c), E^{s u p}\left(c^{\prime}\right)\right) \\
& +\pi_{\text {sub }}^{C} M \operatorname{Sim}_{C}\left(E^{s u b}(c), E^{s u b}\left(c^{\prime}\right)\right) \\
& +\pi_{\text {sib }}^{C} M \operatorname{Sim}_{C}\left(E^{s i b}(c), E^{s i b}\left(c^{\prime}\right)\right)
\end{aligned}
$$

where the set function $\mathrm{MSim}_{C}$ computes the similarity of two entity collections.

As a matter of fact, a distance between two set of classes can be established by finding a maximal matching maximizing the summed similarity between the classes:

$$
\operatorname{MSim}_{C}\left(S, S^{\prime}\right)=\frac{\max \left(\sum_{\left\langle c, c^{\prime}\right\rangle \in \operatorname{Pairing}\left(S, S^{\prime}\right)} \operatorname{Sim}_{C}\left(c, c^{\prime}\right)\right)}{\max \left(|S|,\left|S^{\prime}\right|\right)}
$$

in which Pairing provides a matching of the two set of classes. The OLA algorithm is an iterative algorithm that compute this similarity [10]. This measure is normalized because, if $\mathrm{Sim}_{C}$ is normalized, the divisor is always greater or equal to the dividend.

A normalized similarity measure can be turned into a distance measure by taking its complement to $1\left(E_{C}^{\text {dist }}(x, y)=1-\operatorname{Sim}_{C}(x, y)\right)$. Such a distance introduces a new relation $E_{C}^{d i s t}$ in the concept network $\mathcal{C}$. This relation indeed defines a distance network as introduced above.

\subsection{From Concept Similarity to Ontology Similarity}

Once such a distance has been introduced at the concept level, it can be used for computing a new distance at the ontology level. Again, a distance between two ontologies can be established by finding a maximal matching maximizing similarity between the elements of this ontology and computing a global measure which can be further normalized:

Definition 3 (Ontology distance). Given a set of ontologies $N_{O}$, a set of entities $N_{C}$ provided with a distance function $E_{C}^{\text {dist }}: N_{C} \times N_{C} \longrightarrow\left[\begin{array}{ll}0 & 1\end{array}\right]$ and a relation Defines : $N_{O} \times N_{C}$, the distance function $E_{O}^{\text {dist }}: N_{O} \times N_{O} \longrightarrow\left[\begin{array}{ll}0 & 1\end{array}\right]$ is defined as:

$$
E_{O}^{\text {dist }}\left(o, o^{\prime}\right)=\frac{\max \left(\sum_{\left\langle c, c^{\prime}\right\rangle \in \text { Pairing }\left(\text { Defines }(o), \text { Defines }\left(o^{\prime}\right)\right)} E_{C}^{\text {dist }}\left(c, c^{\prime}\right)\right)}{\max \left(\mid \text { Defines }(o)|,| \text { Defines }\left(o^{\prime}\right) \mid\right)}
$$


Of course, even with these heavy computations, $\forall o \in N_{O}, E_{O}^{\text {dist }}(o, o)=0$.

This is the measure that is used in the OLA algorithm for deciding which alignment is available between two ontologies [10]. However, other distances can be used such as the well known single, average and multiple linkage distances.

This ontology distance introduces a new relation on the ontology layer. This measure provides a good idea of the distances between ontologies. These distances, in turn, provide hints of the difficulty to find an alignment between ontologies. It can be used for choosing to match the closest ontologies with regard to this distance. This can help a newcomer in a community to choose the best contact point: the one with who ease of understanding will be maximized. This will be further developed in Section 5.4 .

\subsection{From Concept Similarity to Alignment}

It can however happen that people have similar but different ontologies. In order for them to exchange their annotations, they use alignments existing within the ontology network. Alignments, in turn, are the results of applying matching algorithms based on the correspondence between ontologies.

As a result, from concept similarity these algorithms will define a new relation $E^{\text {align }}$ at the ontology level.

Definition 4 (Alignment relation). Given a set of ontologies $N_{O}$, a set of entities $N_{C}$ provided with a relation $E_{C}^{\text {dist }}: N_{C} \times N_{C}$, a matching algorithm Match based on $E_{C}^{\text {dist }}$ and a relation Defines : $N_{O} \times N_{C}$, the alignment relation $E^{\text {align }}$ is defined as:

$$
\left\langle o, o^{\prime}\right\rangle \in E^{\text {align }} \text { iff Match }\left(o, o^{\prime}\right) \neq \emptyset
$$

If one has a measure of the difficulty to use an alignment or of its quality, this network can also be turned into a distance network on which all these measures can be performed.

This new relation in the ontology layer allows the agents to choose the ontology that they will align with first. Indeed, the ontologies with maximal hub centrality and closeness are those for which the benefit to align to will be the highest because they are aligned with more ontologies at the ontology level. In the P2P sharing application, choosing such an ontology will bring the maximum answers to queries. For example, in the concept layer of Fig. 1 two alignments between i) po Arun and po Jason and ii) posebastien and po Jason enable Arun and Sebastien to share information, even though they are not explicitly linked with each other.

This is the occasion to note the difference between the relations in the same network: in the ontology network, the hub ontologies for the import relation are rather complete ontologies that cover many aspects of the domains, while hub ontologies for the $E^{\text {align }}$ relation are those which will offer access to more answers.

Of course, when an alignment exists between all the ontologies used by two peers, there is at least some chance that they can talk to each others. This can be further used in the social network.

\subsection{From Ontology Similarity to People Affinity}

Once these measure on ontologies are obtained, this distance can be further used on the social layer. As we proposed it is possible to think that people using the same ontologies 
should be close to each other. We can consider measuring the affinity between people from the similarity between the ontology they use.

Definition 5 (Affinity). Given a set of people $N_{S}$, a set of ontologies $N_{O}$ provided with a distance $E_{O}^{\text {dist }}: N_{O} \times N_{O} \longrightarrow\left[\begin{array}{ll}0 & 1\end{array}\right]$ and a relation Uses $: N_{S} \times N_{O}$, the affinity is the similarity measure defined as

$$
E^{a f f}\left(p, p^{\prime}\right)=\frac{\max \left(\sum_{\left\langle o, o^{\prime}\right\rangle \in \operatorname{Pairing}\left(U \operatorname{se}(p), U \operatorname{se}\left(p^{\prime}\right)\right)} 1-E_{O}^{\text {dist }}\left(o, o^{\prime}\right)\right)}{\max \left(|U \operatorname{se}(p)|,\left|U s e\left(p^{\prime}\right)\right|\right)}
$$

Since this measure is normalized, it can be again converted to a distance measure through complementation to 1 .

Introducing the distance corresponding to affinity in the social network allows to compute the affinity relationships between people with regard to their knowledge structure. Bottom-up inference from $\mathcal{C}$ allows to find out the semantic relationships between users based on this space.

For completing the $\mathrm{P} 2 \mathrm{P}$ application, the last step consists of identifying the subgroups of users, according to the various social characteristics, as follows:

1. The subgroup whose members are assigned very high semantic authoritative weight (or semantic hub weight) can be identified by comparing the weights computed with Equ. 4. These peers have the social power to control and select semantic information to distribute.

2. The subgroups of people with very similar personal ontologies can be obtained by computing the cohesive subgroup of the $\mathcal{S}$ network using affinity $\left(E^{a f f}\right)$.

3. The subgroup of people which are interested in the same topics extends the previous subgroups, depending on a particular topic. Their members can efficiently share information about that topic.

\section{Experimental Results}

As a first evaluation of our framework, we want to differentiate social affinity $E^{a f f}$ $\left(p, p^{\prime}\right)$ from the previous measures for social features. Our experimentation scenario follows these steps $(i)$ building personal ontologies during photo annotation, $(i i)$ aligning these personal ontologies for measuring social affinity between people, and (iii) discovering the most powerful person for semantic interoperability.

For collecting data, we invited seven members of our team to select a set of photographs and annotate them by using a specific annotation tool (Picster 2 ). Table 2 shows the specification of personal ontologies.

From co-occurrence patterns between the annotated photos, Mika's social centrality $C_{M}$ [3] can be formulated by

$$
C_{M}\left(U_{i}\right)=\frac{\sum \frac{\cap_{k=1, k \neq i}^{|U|}\left(\mathcal{R}_{U_{k}}, \mathcal{R}_{U_{i}}\right)}{\mathcal{R}_{U_{i}}}}{|U|-1}
$$

where $|U|$ is the total number of people in the social network. The results are shown in Table 3 . We found out that the number of annotated resources are barely related to the

\footnotetext{
${ }^{2}$ http://gforge.inria.fr/projects/elster
} 
Table 2. Specification of personal ontologies as test bed

\begin{tabular}{r|ccccccc}
\hline & AS & AZ & FAK & JE & JJ & JP & SL \\
\hline Number of annotated photographs $\left(\mathcal{R}_{\text {User }}\right)$ & 47 & 47 & 37 & 49 & 47 & 30 & 25 \\
Number of used ontologies $\left(\mathcal{O}_{\text {User }}\right)$ & 3 & 5 & 2 & 6 & 1 & 1 & 2 \\
\hline
\end{tabular}

Table 3. Experimental results of a) closeness and centrality by co-occurrence patterns b) people affinity $E^{a f f}$ and centrality in the semantic social network

\begin{tabular}{c|ccccccc|c|c}
\hline (a/b) & AS & AZ & FAK & JE & JJ & JP & SL & $C_{M}$ & $C_{a f f}$ \\
\hline AS & - & $0.98 / 0.65$ & $0.62 / 0.33$ & $0.94 / 0.73$ & $1.00 / 0.26$ & $0.60 / 0.32$ & $0.23 / 0.62$ & 0.73 & 0.49 \\
AZ & 0.98 & - & $0.62 / 0.49$ & $\mathbf{0 . 9 4 / 0 . 8 2 5}$ & $0.98 / 0.31$ & $0.62 / 0.3$ & $0.26 / 0.52$ & 0.73 & 0.52 \\
FAK & 0.78 & 0.78 & - & $0.70 / 0.57$ & $0.78 / 0.28$ & $0.54 / 0.22$ & $0.30 / 0.32$ & 0.65 & 0.37 \\
JE & 0.90 & $\mathbf{0 . 9 0}$ & 0.53 & - & $0.90 / 0.46$ & $0.57 / 0.49$ & $0.16 / 0.75$ & 0.66 & $\mathbf{0 . 6 4}$ \\
JJ & 1.00 & 0.98 & 0.62 & 0.94 & - & $0.60 / 0.72$ & $0.23 / 0.39$ & 0.73 & 0.40 \\
JP & 0.93 & 0.97 & 0.67 & 0.93 & 0.93 & - & $0.13 / 0.51$ & $\mathbf{0 . 7 6}$ & 0.43 \\
SL & 0.44 & 0.48 & 0.44 & 0.32 & 0.44 & 0.16 & - & 0.38 & 0.52 \\
\hline
\end{tabular}

social centrality. SL annotated the least number of resources, so that his centrality also lowest among people. But, even though JE's annotations were the largest one, JP has shown the most powerful centrality.

We measured semantic affinity on the semantic social network (Eq.8). For doing so, the ontology distances $E_{O}^{\text {dist }}$ between personal ontologies are measured. We used string edit distance between class labels. For instance, $E_{O}^{\text {dist }}$ between JE and AZ is shown in Table4 Then, we can measure $E^{a f f}(\mathrm{AZ}, \mathrm{JE})=\frac{\max \left(\sum_{\left\langle o, o^{\prime}\right\rangle} 1-E_{O}^{\text {dist }}\left(o, o^{\prime}\right)\right)}{\max (5,6)}=\frac{4.95}{6}=$ 0.82 where ontology distance between non aligned ontologies ('-') are regarded as 1 . We computed this for all pairs of people on the social network, as shown in Table 3 . The matrix for social affinity is symmetric. We found out that the number of ontologies are playing an important role in social affinity. JE has shown the highest centrality in the given social network, while JP annotated the most common resource with other people. This means that collaborations can be effectively be provided with JE. Meanwhile, $E^{a f f}(\mathrm{JJ}, \mathrm{JP})$ was relatively high $(0.72)$. We found that they were using the same large ontology (i.e., SUMO). So the number of found correspondences where very high. To deal with this problem, we have to consider preprocessing personal ontologies.

Table 4. Ontology distance $E_{O}^{\text {dist }}$ between JE and AZ; Mark '-' means no alignments between two ontologies

\begin{tabular}{c|cccccc}
\hline & JE_foaf.owl & JE_Meteo.owl & JE_Picster.owl & JE_space.owl & JE_UrbanLand.owl & JE_World.owl \\
\hline az_support-ontology.owl & 0.03 & - & - & 0.17 & - & - \\
az_hasSupplyLineOnt.owl & 0.46 & - & 0.09 & 0.05 & 0.04 & 0.49 \\
az_office.owl & 0.47 & - & 0.04 & 0.05 & 0.06 & 0.04 \\
az_people+petsB.owl & 0.06 & - & - & 0.16 & - & - \\
az_space-basic.owl & 0.18 & - & - & 0.5 & - & 0.01 \\
\hline
\end{tabular}


Another issue is the discovery of potential collaborators (or like-minded people) by comparing the social distances in Table 1 While the social distance between AS and SL is 3 on the social layer of Figure 1 (i.e., 1. once normalized), their social affinity is measured as 0.62 which is relatively high (the corresponding distance would be .38). We can expect that they can share common interests.

\section{Related Work}

Many semantic systems on distributed environments like P2P networks have been introduced to efficiently share information and knowledge between heterogeneous sources. Some have studied the relevance of peers (or users) by analyzing topology and interactions like message passing. In [11], for selecting the expert peers, semantic topology analysis is exploited. But they make assumptions that every peers have to use the same ontology for calculating semantic similarity. Practically, [12] applies the similar idea to multi-agent architecture, and as an example of application, Jung has introduced a social communication framework for collaborative web browsing [13]. Meanwhile, Alani and colleagues introduced a system, called Ontocopi, for ontology-based network analysis (ONA) [14]. This system can identify the communities by using informal data.

More closely related to this work, Mika proposes a three-layered space which is composed of a social network and a knowledge network relying on concepts and instances [15]. However, the knowledge network is simply based on sets for co-occurrence analysis, and in this networks, the super/sub-relationships are retrieved based on statistical overlapping. This approach does not deeply consider semantic relationships between concepts and ontologies, so it is hard to use the structure of knowledge for structuring the social network.

For measuring the relevance of ontologies, in [4], the AKTiveRank system ranks the ontologies applying a number of classical metrics such as class match, centrality, density, and semantic similarity. In [16], network analysis methods (in particular, Hermitian matrices-based eigensystem analysis) are used for analyzing ontologies as concept graphs in the same way social network are analyzed. This is used for contrasting the "style" of ontologies but not for social networking, though this last application could be investigated.

\section{Concluding Remarks and Future Work}

We have focused on using the structure of knowledge used by people in order to extract meaningful relations at the social level. Moreover, the extraction of these new relations is used to further improve the collaborative sharing and exploitation of this knowledge.

We propose a three-layered architecture for constructing semantic social network, which is composed of a social layer, an ontology layer, and a concept layer. This space not only supports the relations within a layer, but also the propagation of relations between layers. We have provided the principles for extracting similarity between concepts and propagating this similarity to a distance and an alignment relation between ontologies. This distance relation can be used for discovering affinity in the social network. In return, users can take advantage of these newly established relations to find 
people closer to them on the basis of the structure of their knowledge. For that purpose, we have only used classical SNA measures (that we extended to distance networks).

The basic assumption of this work is that these newly discovered relations between people will facilitate mutual understanding as well as ontology matching and resource sharing. This remains to be demonstrated experimentally. To that extent we are developing and experimenting the presented P2P sharing system. We will make measure and generate alignment and ask users to rate and correct the provided alignments.

There remains important issues to be investigated: all these networks are not equal and their exploitation with classical SNA tools can be meaningless (in the same sense that considering the "loves" and "hates" relations as the same would lead to problems). It is thus important to characterize the various relations that were provided with regard to the measures that can be used on them. We also plan to extend this work by defining and extracting meaningful (and useful) clusters among people. We expect to apply this information to build social and semantic grid environment [17]. Moreover, the cost of computing these networks can become important. We have not considered this issue here because most of the given measures are examples, but this can become an important factor if the networks and measures have to be computed on-line.

\section{References}

1. Wasserman, S., Faust, K.: Social network analysis: methods and applications. Cambridge University Press, Cambridge (UK) (1994)

2. Kleinberg, J.M.: Authoritative sources in a hyperlinked environment. J. of the ACM 46(5) (1999) 604-632

3. Mika, P.: Ontologies are us: A unified model of social networks and semantics. In Gil, Y., Motta, E., Benjamins, V.R., Musen, M.A., eds.: Proc. of the 4th Int. Semantic Web Conf. Volume 3729 of Lecture Notes in Computer Science., Springer (2005) 522-536

4. Alani, H., Brewster, C.: Ontology ranking based on the analysis of concept structures. In: Proc. of the 3rd Int. Conf. on Knowledge capture (K-CAP '05), New York, NY, USA, ACM Press (2005) 51-58

5. Broekstra, J., Ehrig, M., Haase, P., van Harmelen, F., Menken, M., Mika, P., Schnizler, B., Siebes, R.: Bibster - a semantics-based bibliographic peer-to-peer system. In: Proc. of the 2nd Work. on Semantics in Peer-to-Peer and Grid Computing (SemPGRID '04), New York, USA (2004) 3-22

6. Euzenat, J.: Alignment infrastructure for ontology mediation and other applications. In: Proc. of the 1st Int. Work. on Mediation in Semantic Web Services, Amsterdam (NL). (2005) 81-95

7. Freeman, L.: Centrality in social networks: Conceptual clarification. Social Networks $\mathbf{1}$ (1979) 215-239

8. Fortunato, S., Latora, V., Marchiori, M.: Method to find community structures based on information centrality. Physical review E. 70 (2004) 056104

9. Shvaiko, P., Euzenat, J.: A survey of schema-based matching approaches. J. on data semantics 4 (2005) 146-171

10. Euzenat, J., Valtchev, P.: Similarity-based ontology alignment in OWL-Lite. In de Mántaras, R.L., Saitta, L., eds.: Proc. of the 16th Euro. Conf. on Artificial Intelligence, IOS Press (2004) 333-337 
11. Haase, P., Siebes, R., van Harmelen, F.: Peer selection in peer-to-peer networks with semantic topologies. In Bouzeghoub, M., ed.: Proc. of the Int. Conf. on Semantics in a Networked World (ICNSW'04). Volume 3226 of Lecture Notes in Computer Science., Springer-Verlag (2004) 108-125

12. Valencia, E., Sansonnet, J.P.: Building semantic channels between heterogeneous agents with topological tools. In: Proc. of the 2nd Euro. Work. on Multi-Agent Systems. (2004)

13. Jung, J.J.: Collaborative web browsing based on semantic extraction of user interests with bookmarks. J. of Universal Computer Science 11(2) (2005) 213-228

14. Alani, H., Dasmahapatra, S., O'Hara, K., Shadbolt, N.: Identifying communities of practice through ontology network analysis. IEEE Intelligent Systems 18(2) (2003) 18-25

15. Staab, S., Domingos, P., Mika, P., Golbeck, J., Ding, L., Finin, T., Joshi, A., Nowak, A., Vallacher, R.R.: Social networks applied. IEEE Intelligent Systems 20(1) (2005) 80-93

16. Hoser, B., Hotho, A., Jäschke, R., Schmitz, C., Stumme, G.: Semantic network analysis of ontologies. In: Proc. of the 3rd Euro. Semantic Web Conf. (ESWC). Volume 4011 of Lecture Notes in Computer Science (2006) 514-529

17. Jung, J.J., Ha, I., Jo, G.: BlogGrid: Towards and efficient information pushing service on blogspace. In Zhung, H., Fox, G., eds.: Proc. of the Int. Conf. Grid and Cooperative Computing (GCC 2005). Volume 3795 of Lecture Notes in Computer Science., Springer-Verlag (2005) 178-183 